\title{
Perspectivas sociológicas en el abordaje de las trayectorias: un análisis sobre los usos, significados y potencialidades de una aproximación controversial
}

EUGENIA ROBERTI"

\section{Resumen}

El objetivo del artículo es estudiar los aportes que brinda la perspectiva de las trayectorias. Desde este lugar, realizamos una reflexión crítica junto a una sistematización teórica de aquellas investigaciones que nos aproximen a comprender los supuestos que se esconden tras estos estudios. La primera sección indaga sobre los orígenes del uso de biografías en la investigación social; nos centramos en los enfoques europeo y norteamericano para vislumbrar los distintos ámbitos de producción en donde surgieron los fundamentos que encuadran y dan sentido al análisis de trayectorias. Un segundo apartado elabora una construcción teóricometodológica que problematiza el estudio de las trayectorias, proponiendo un marco analítico para su comprensión, a partir de discutir diferentes perspectivas y conceptualizaciones. En este punto, se hace especial hincapié en las potencialidades de los estudios con trayectorias laborales. Para finalizar, buscamos recapitular los aportes que la perspectiva escogida brinda a la investigación social, posibilitando aprehender los fenómenos sociales desde su complejidad analítica, al atender la dimensión objetiva y subjetiva en su articulación espacio-temporal.**

Palabras clave: Investigación social. Estudios biográficos. Trayectoria. Espacio Tiempo

\footnotetext{
* Universidad Nacional de La Plata, Argentina

** Parte de este artículo ha sido elaborada en el contexto de INCASI Network, un proyecto europeo que ha recibido financiación del programa de investigación e innovación Horizon 2020 de la Unión Europea, bajo la sección Marie Skłodowska-Curie GA no 691004 y coordinado por Dr. Pedro López-Roldán. Este artículo refleja únicamente la opinión del autor y la Agencia no es responsable de ningún uso que pueda hacerse de la información que contiene
} 


\title{
Sociological perspectives in the trajectories framework: an analysis on the uses, meanings, and potentialities of a controversial approach
}

\begin{abstract}
This article aims to analyse the contributions provided by the trajectories approach. Thence, a critical reflection is carried out together with a theoretical systematization of researches that may help to understand the underlying assumptions of these studies. The first section looks into the origins of the use of biographies in social research, focusing on European and North American approaches to look at the different areas where the foundations that frame and give meaning to the analysis of trajectories arose. A second section builds a theoretical-methodological framework that problematizes the study of trajectories, proposing an analytical framework for its understanding, based on a discussion of different perspectives and conceptualizations. Special emphasis is placed on the potential of studies on labour trajectories. Finally, we seek to recapitulate the contributions that the chosen perspective provides to social research, enabling to grasp the analytical complexity of social phenomena, by dealing with both the objective and subjective dimensions in their spatio-temporal articulation.
\end{abstract}

Keywords: Social research. Biographical studies. Trajectories. Space-Time. 


\section{Introducción}

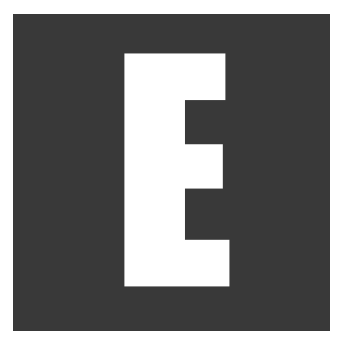

Toda tentativa de periodización de una trayectoria biográfica (o de un haz de trayectorias) debe considerar dos órdenes de acontecimientos distintos, pero relativamente dependientes: «eventos individuales» que conforman los diferentes recorridos constitutivos de una trayectoria biográfica (pero cuyas regularidades reflejan la historia de las estructuras sociales); «eventos históricos» que pautan el devenir de las estructuras sociales (y donde las trayectorias individuales dejan sus huellas) (Mauger, 1989, traducción propia)

en que - desde nuestra mirada - el campo de estudios biográficos se constituye como telón de fondo en los análisis de trayectorias. Nos centramos en los enfoques europeo y norteamericano para vislumbrar los distintos ámbitos de producción en donde surgieron los supuestos que se esconden tras estos estudios.

En segundo lugar, realizamos un recorrido por las obras clásicas y las principales investigaciones que tanto en América Latina como en Argentina han utilizado este enfoque; en especial, analizamos la esfera laboral como dispositivo particular de indagación en el estudio de las trayectorias. Por último, señalamos las potencialidades del estudio de trayectorias en el campo de la investigación social, centrándonos en tres aspectos fundamentales de esta perspectiva teórico-metodológica: la imbricación de niveles objetivos-subjetivos, la conjunción de temporalidades y la relevancia de la dimensión espacial. 
Para iniciar este recorrido, partimos de los siguientes interrogantes: ¿Desde qué orientación o marco referencial abordar el estudio de las trayectorias? ¿Cuáles son los aportes de esta perspectiva teórico-metodológica a la investigación social? ¿Cómo se introduce la dimensión temporal y espacial en el análisis de trayectorias? Responder estos interrogantes, y posicionarnos frente a ellos, nos permitirá presentar nuestra propia perspectiva de análisis para el abordaje de trayectorias.

\section{El campo de estudios biográficos como marco referencial de las trayectorias}

Los estudios biográficos se constituyen como un campo de investigación en las Ciencias Sociales a principios del siglo XX. Sus orígenes se asocian a los numerosos análisis que, en el marco de la Escuela de Chicago, proliferaron a partir de la década del '20 en base a estudios de caso'. Sin embargo, es en el ámbito de la antropología norteamericana donde el método biográfico alcanza su mayor desarrollo y expansión.

Con posterioridad a la Segunda Guerra Mundial, tanto en la sociología como en la antropología, las historias de vida fueron relegadas como instrumento auxiliar y de apoyo en las investigaciones sociales, recibiendo críticas en relación a su escasa cientificidad, por no satisfacer los requerimientos de representatividad y validez (Piña, 1986). La primacía de la premisa epistemológica de objetividad promulgó la utilización de métodos cuantitativos: el survey research. El surgimiento de los Estados Unidos como potencia mundial conllevó a la hegemonía de la sociología norteamericana - con sus pilares en el método de encuestas y el funcionalismo

\footnotetext{
${ }^{1}$ Entre estos estudios se pueden mencionar los trabajos de W.I. Thomas y F. Znanniecki, The Polish Peasant in Europe and America; C. Shaw, The jack roller, the natural history of a delinquent career, Brothers in crime; E. Sutherland, The professional thief; H. Macgill Hughes, The fantastics lodge, entre otros.
} 
parsoniano - sobre otras formas de observación y de teorización (Bertaux, 1999).

Hubo que esperar hasta fines de la década del '60 para que, en un contexto de revalorización de la metodología cualitativa, la modalidad de investigación con historias de vida experimentara un vigoroso y progresivo resurgimiento, volviendo a ocupar un lugar destacado en la investigación epistemológica, teórica y metodológica de las Ciencias Sociales. La consolidación del campo de estudios biográficos se efectuó, así, en el marco de una multiplicidad de disciplinas que tomaron como objeto de estudio los relatos e historias de vida, las narrativas autobiográficas, la historia oral y, en estrecha relación con nuestra investigación, los análisis de trayectorias (Muñiz Terra; Roberti, 2013).

Desde la sociología, la especificidad del estudio con biografías se vinculó con el análisis de las experiencias que una persona construye a lo largo de su vida, en la cual la narración del propio sujeto, entre otros documentos biográficos, adquiere un lugar central. Esta perspectiva busca aprehender los acontecimientos ocurridos en el transcurso de las vidas individuales, como el resultado de una historia que entrelaza experiencias pasadas y expectativas futuras. Por esta razón, no toma en cuenta un momento particular de la vida de la persona, sino que intenta reconstruir su trayectoria a lo largo del tiempo o en un período determinado. El investigador que reconstruye historias de vida indagará sobre los sentidos subjetivos, significados y representaciones que un individuo elabora acerca de su historia personal.

Otra de las principales preocupaciones de la investigación sociológica, que trasluce las potencialidades del uso de biografías, ha sido el análisis de la relación entre individuo y sociedad. Pese a que la perspectiva biográfica intenta otorgar una instancia privilegiada a las vivencias y experiencias de un sujeto, el planteo no se reduce a examinar la singu- 
laridad de una vida, sino a entrelazar las experiencias particulares con las transformaciones de la sociedad en general; permitiendo aprehender aquella biografía como el reflejo de una época, normas sociales y valores de un período determinado (Pujadas Muñoz, 1992; Hareven; de Gruyere, 1999). De este modo, se plantea una mediación entre la historia individual y la historia social: "ni la vida de un individuo ni la historia de una sociedad puede entenderse sin entender ambas cosas" (Wright Mills, 1994, p. 23). ${ }^{2}$

En este marco, los estudios biográficos se muestran fértiles para estudiar la estructura social o, desde una mirada opuesta, el cambio social. No se trata sólo de aprehender la estructura a partir de la singularidad de una vida, sino también, lo que ilumina el análisis de las secuencias de cambio del curso vital sobre los procesos históricos de la sociedad (Balán; Jelin, 1979). La interacción entre contexto sociohistórico e historia personal se vislumbra al analizar las consecuencias de ciertos acontecimientos sobre la continuidad o variación en la dirección de las trayectorias vitales. En este sentido, la incorporación de la dimensión temporal permite un análisis de los procesos de cambio a nivel individual y colectivo (familias, cohortes), al examinar las posiciones ocupadas en distintos momentos del ciclo de vida (Jelin, 2006).

Como apuntamos precedentemente, a fines de los años '60, el uso de biografías adquirió un impulso renovado. A su interior, el mismo se manifestó en el desarrollo de dos corrientes: el Curso de Vida en Estados Unidos (Elder, 1993, 2001; Hareven, 1994; Saraceno, 2005) y el Enfoque Biográfico en Europa (Pujadas Muñoz, 1992; Ferrarotti, 1988, 1991,

\footnotetext{
2 Referentes clásicos del campo biográfico europeo, como Bertaux (1997) y Ferrarotti (1993), destacan la importancia de la perspectiva del individuo como punto de observación de la sociedad. Para estos autores, el uso de historias de vida es un intento de lectura de lo social desde los sujetos; lo que una vida singular trasluce respecto a un contexto social más amplio como puede ser, un momento histórico, una cultura o un grupo social determinado.
} 
1993; Bertaux, 1997, 1999). ${ }^{3}$ El presente artículo considera similares los fundamentos analíticos de ambas corrientes. En términos generales, estas perspectivas buscan dar respuesta al problema de la articulación de lo individual con lo colectivo, investigando las mediaciones entre la estructura social y la biografía personal (Roberti, 2015).

En el Enfoque Biográfico y en el paradigma del Curso de Vida encontramos los supuestos epistemológicos, teóricos y metodológicos que enmarcan y dan sentido a los estudios con trayectorias. Al trascender los dilemas clásicos del pensamiento social - sociedad/individuo; estructura/ acción; objetividad/subjetividad - estas orientaciones permiten analizar la realidad social en su complejidad analítica. De este modo, la perspectiva biográfica es un intento de superar la falsa oposición entre las perspectivas objetivistas y subjetivistas, estableciendo una articulación entre ambas dimensiones de lo social: atiende a los determinantes sociales en el estudio concreto de una vida como al trabajo que el propio individuo realiza para convertirse en sujeto de la historia, intentando orientar su curso y otorgarle un sentido.

La perspectiva del Curso de Vida se origina durante los años '70 en los Estados Unidos, como una propuesta conjunta de diversas disciplinas dentro de las Ciencias Sociales - sociología, historia, psicología y demografía. Se presenta, de este modo, como un enfoque interdisciplinario que toma como unidad de análisis el curso de vida de un individuo. El

\footnotetext{
3 Para una caracterización pormenorizada del Enfoque Biográfico en Europa, véase: Muñiz Terra, 2009. En esta instancia, cabe resaltar que lo biográfico ha sido concebido de distinta manera en la investigación sociológica europea. Los estudios a partir de relatos e historias de vida han adoptado, de este modo, una pluralidad de contenidos. Habitualmente, las historias de vida se asocian con una de las tantas técnicas que utilizan los cientistas sociales para abordar una problemática en estudio (Pujadas Muñoz, 1992). Sin embargo, otros autores, como Bertaux (1997), recurren a la noción de métodos biográficos, dilucidando en ella una aproximación metodológica más que un mero instrumento de recolección de datos. Para el sociólogo italiano Ferrarotti $(1988,1991)$, en cambio, la historia de vida no es un método o una técnica más, sino una perspectiva de análisis única que permite descubrir lo cotidiano, las prácticas de vida dejadas de lado o ignoradas por las miradas dominantes, la historia de y desde los de abajo.
} 
sociólogo Glen Elder, uno de los principales referentes teóricos de esta corriente, se introduce al interior del debate de los estudios con biografías, concibiendo al Curso de Vida como un "paradigma emergente" (Elder, 1994) que busca incorporar la dimensión cualitativa del tiempo en la investigación. Retomando las ideas del autor, se define al curso de vida como "una secuencia de eventos y roles sociales, graduados por la edad, que están incrustados en la estructura social y el cambio histórico" (Blanco; Pacheco, 2003, p. 162).

Este paradigma se asienta en cinco postulados, que reflejan la modalidad y especificidad de su abordaje analítico, los cuales conforman nuestro marco de interpretación subyacente para el análisis de las trayectorias (véase: Blanco; Pacheco, 2003; Elder et al, 2003; Castro; Gandini, 2006; Longo, 2010).

Principio de desarrollo a lo largo del tiempo: para la comprensión de las biografías es necesaria una perspectiva a largo plazo que permita un análisis relacional entre cambio social y desarrollo individual. En otras palabras, que posibilite dar cuenta del interjuego entre la vida individual y el tiempo histórico-social.

Principio de tiempo y lugar: el curso de vida de los sujetos está incrustado y es moldeado por las diversas escalas de temporalidad y los distintos lugares que experimenta cada persona. La importancia de tomar en consideración la dimensión espaciotemporal radica en que permite ubicar al individuo en el contexto en el que desarrolla su biografía.

Principio de timing: el impacto de una transición o un evento contingente en el desarrollo humano está asociado al período en el que sucede. La incidencia de un acontecimiento tendrá diversas repercusiones de acuerdo al momento de la vida de un individuo y sus circunstancias.

Principio de vidas interconectadas (linked lives): una vida no se constituye aisladamente. Razón por la cual, es importante considerar los 
entornos en los que un individuo se desenvuelve y las relaciones en las que se halla inmerso. A causa de esta interdependencia, las transiciones que se originan en la vida de una persona pueden influenciar el orden y la dirección de las trayectorias de otros sujetos de su medio.

Principio de libre albedrío: al interior de una estructura de oportunidades que implica limitaciones provenientes de las circunstancias histórico-sociales, los individuos hacen elecciones y llevan a cabo acciones construyendo, de esta manera, su propio curso de vida.

El paradigma del Curso de Vida trabaja con tres herramientas conceptuales y metodológicas fundamentales: la trayectoria, la transición y el turning point. Estas categorías, muchas veces mal asimiladas como equivalentes, conforman los instrumentos analíticos del enfoque.

El concepto de trayectoria se refiere "a una línea de vida o carrera, a un camino a lo largo de toda la vida, que puede variar y cambiar en dirección, grado y proporción" (Elder, 1991, p. 63 citado por Blanco; Pacheco, 2003, p. 163). El curso de vida de los sujetos es el resultado del entrelazamiento de múltiples trayectorias que representan diversas dimensiones - trabajo, escolaridad, vida reproductiva, migración, etc. - en las que una persona se desenvuelve a lo largo de su vida, conformando su articulación la "trayectoria vital" de un sujeto (Blanco; Pacheco, 2003; Castro; Gandini, 2006). Si bien en la conformación del curso de vida intervienen múltiples trayectorias, alguna de ellas funcionará como eje o hilo conductor de las demás (Blanco, 2002). Por consiguiente, las trayectorias tomarán una importancia diferencial según el momento de la vida del sujeto. El recorrido biográfico estará constituido, así, por las imbricaciones entre las diversas esferas que, en el juego de sus interdependencias, dan forma al curso de vida.

Otra de las dimensiones analíticas fundamentales del paradigma del Curso de Vida es el concepto de transición, el cual da forma y sentido a 
la trayectoria, al ser constitutiva de ésta; hace referencia a eventos específicos en ciertos momentos de la vida, que marcan cambios de estado, posición o situación (Blanco; Pacheco, 2003). Las transiciones están contenidas en las trayectorias: mientras la primera representa el pasaje de un estado a otro (de la juventud a la adultez, del ciclo básico al superior, del desempleo a la actividad, etc.), la trayectoria se define como el conjunto de esos pasajes y estados; aquellas secuencias de transiciones y posiciones en un período determinado (Gautié, 2003).

Para el enfoque del Curso de Vida, "la trayectoria no supone alguna secuencia en particular ni determinada velocidad en el proceso de tránsito" (Blanco; Pacheco, 2003, p. 163). Más aún, rechaza la homogeneidad y la linealidad temporal asumiendo una concepción multidimensional del tiempo (Blanco, 2002). Los estados específicos en ciertos momentos de la vida, a los que hace alusión el concepto de transición, no son absolutamente previsibles ni se encuentran necesariamente predeterminados. Si bien, ciertas transiciones se hallan institucionalizadas en una sociedad, variando de acuerdo al período histórico y grupo social de pertenencia. Al punto que, el orden de los diversos estados y sus transiciones en muchas ocasiones responden a las expectativas sociales, culturales e institucionales en torno a la edad (Castro; Gandini, 2006).

Por último, la dimensión temporal se despliega como la posibilidad de inscribir lo nuevo (el evento que irrumpe) al interior de una biografía. Precisamente, la noción de turning point - punto de inflexión - hace referencia a "momentos especialmente significativos de cambio; se trata de eventos o transiciones que provocan fuertes modificaciones que, a su vez, se traducen en virajes en la dirección del curso de vida" (Blanco; Pacheco, 2003, p. 163). Cabe destacar, entonces que el análisis a partir de este paradigma incorpora aquellos períodos de ruptura y reorientación en la vida de una persona. Estos momentos de inflexión "representan un cambio en 
la dirección del curso de vida en relación a la trayectoria pasada y tienen un impacto en las probabilidades de los destinos de vida futura" (Gobtlb; Wheaton, 1997, p. 5).

Estos períodos de cambio encuentran sus orígenes en puntos de inflexión históricos (como puede ser una crisis económica, una guerra, una perturbación política, etc.) o personales, ligados a transformaciones en el curso vital de un individuo (en relación a su residencia, estado civil, posición ocupacional, etc.). Por esta razón, para el paradigma del Curso de Vida la trayectoria se torna inteligible cuando se articulan los hechos histórico-sociales, en conjunto con las etapas cronológicas y los entornos sociales donde se desarrolla. En suma, dicha perspectiva nos permite pensar el tiempo desde la vida de los propios individuos en articulación con los procesos sociales más amplios, que se encuentran mediatizados por la posición del sujeto en la estructura social.

\section{Las trayectorias como dispositivo de indagación:} un análisis de sus potencialidades para la investigación social

Como perspectiva de análisis, el estudio de las trayectorias es un campo de investigación relativamente novedoso en América Latina, que en las últimas décadas ha ido adquiriendo un lugar importante en el ámbito de las Ciencias Sociales. Durante los años '70 surgen en México los primeros estudios sobre trayectorias, constituyéndose en un hito el trabajo de Balán, Browning y Jelin (1977) El hombre en una sociedad en desarrollo: movilidad geográfica y social en Monterrey. La influencia del paradigma del Curso de Vida alcanza su mayor expansión en este país, recibiendo un impulso destacado en el marco de los estudios sociodemográficos (Tuirán, 1999; Oliveira; Ariza, 2001; Blanco; Pacheco, 2003; Castro; Gandini, 2006). En particular, en sus orígenes la perspectiva de 
las trayectorias se inscribió en el campo más amplio de los análisis sobre movilidad socio-ocupacional, atendiendo dicha problemática desde una metodología cuantitativa.

En la última década del siglo XX, el estudio de trayectorias se comienza a desarrollar en Brasil, de la mano de las preocupaciones por la reestructuración económica y productiva de los años '90, que impacta en la subjetividad e itinerarios de los trabajadores industriales (Cabanes, 1998; Bueno Fartes, 2001; Moreira Cardoso et al, 2001). Hubo que esperar hasta comienzos del nuevo siglo para que surgiera en Chile un campo de estudio abocado al análisis con trayectorias (Guzmán et al, 1999; Márquez, 2001; Irrazabal; Oyarzún, 2003; Mauro, 2004). Es el caso también de Argentina, como veremos a continuación, donde un conjunto de investigaciones recientes utiliza esta perspectiva teórico-metodológica para el análisis de diversos fenómenos sociales. La particularidad de este origen tardío concede un lugar más importante a la metodología cualitativa y habilita una mayor influencia de las aproximaciones europeas.

Como dispositivo particular de indagación, la perspectiva de las trayectorias laborales en Argentina ha recibido un impulso renovado en las últimas décadas. En términos generales, dichos estudios se han focalizado en reconstruir las secuencias de actividad o categorías ocupacionales a lo largo de un período determinado. Tales investigaciones han abordado diversas temáticas y segmentos ocupacionales: existen estudios sobre grupos profesionales (Panaia, 2001; Testa; Figari, 2005; Berdaguer; Zarauza, 2011) y poblaciones obreras (Dávolos, 2001; Frassa, 2005; Muñiz Terra, 2009), algunos de los cuales centran la mirada en cómo los procesos de reestructuración productiva incidieron en los trayectos de sus trabajadores. Otros trabajos analizan la inserción ocupacional de jóvenes (Freytes Frey, 2007; Jacinto, 2010; Longo, 2010); en este punto, son conocidos los estudios sobre la transición entre la educación y el trabajo (Montes, 2009; 
Otero, 2009), donde se destacan los clásicos abordajes de "seguimiento de egresados" (Filmus et al, 2003; Sendón, 2005; Miranda et al, 2014). Por último, se encuentran investigaciones que abordan la problemática de la exclusión social (Forni; Roldán, 1996; Freidin, 1996; Salvia; Chávez Molina, 2007) y estudios sobre las trayectorias laborales de mujeres, que vislumbran la discriminación e inequidad de género en el mercado laboral (Cragnolino, 2003; Cutuli, 2008; Millenaar, 2012).

En correspondencia con esta pluralidad de núcleos temáticos, la perspectiva de la trayectoria ha sido concebida y utilizada de manera ampliamente heterogénea. Los disímiles usos y aplicaciones desarrolladas en las investigaciones reflejan tanto la multiplicidad de conceptualizaciones empleadas en el abordaje de diversas problemáticas como también las distintas estrategias metodológicas presentes en cada análisis. No obstante, pese a esta diversidad, dicha perspectiva teórico-metodológica se inscribe en una clásica discusión de la tradición sociológica, expresada en la dicotomía individuo-sociedad, subjetivismo-objetivismo, estructura-acción; temática que recorre las obras de referentes indiscutidos del campo disciplinar: desde Marx, Durkheim y Weber hasta, más contemporáneamente, Bourdieu, Giddens y Lahire.

Ubicado en este clásico dilema sociológico, el estudio de trayectorias se constituye como un campo de análisis en disputa, que recurre a diversas orientaciones teóricas para su formulación. Numerosas investigaciones sociales han abordado la problemática de la constitución de las trayectorias desde enfoques estructuralistas (Godard, 1996; Bourdieu, 1997b; Coninck; Godard, 1998; Casal et al, 2006), privilegiando el análisis de las posiciones sucesivas que las personas ocupan a lo largo de su vida o durante un período determinado. Esta perspectiva presta atención al eslabonamiento de causalidades, al orden y a la duración de los acontecimientos, concebidos como externos o condicionantes de la acción 
social. Frente a esta "ilusión objetivista" (Clot, 2011), un conjunto de investigaciones ha destacado la necesidad de atender el carácter subjetivo de las trayectorias, tomando como eje analítico el particular entramado de experiencias personales. Desde este enfoque, se intenta reconstruir el encadenamiento de sucesos a partir del proceso decisorio llevado a cabo por los sujetos en el transcurso de su recorrido vital (Kohli, 2005; Saraceno, 2005; Pais, 2007; Gil Calvo, 2009).

Una larga tradición sociológica se apoya en la primera tendencia teórica, cuyo referente indiscutido ha sido Pierre Bourdieu. Para entender el concepto de trayectoria inscripto en la teoría bourdiana, es necesario analizar la oposición que el autor establece con la noción de biografía. Pretendiendo tomar distancia de las explicaciones subjetivistas, que comprenden la acción como la realización de una intención consciente, el sociólogo francés realiza un cuestionamiento a aquellas perspectivas que conciben la vida de un individuo como un conjunto coherente y orientado de sucesos, que puede aprehenderse como expresión de un sentido último u originario. Relatar la vida como una historia, como la narración de una secuencia lógica de acontecimientos, no es más que una ilusión, la creación artificial de sentido (Bourdieu, 1997b). En la construcción del relato biográfico, el investigador debe evitar suponer que existe un hilo conductor que atraviesa la vida del sujeto desde sus orígenes.

Detrás de esta crítica a la perspectiva subjetivista, la noción de trayectoria que elabora Bourdieu hace referencia a la "serie de las posiciones sucesivamente ocupadas por un mismo agente (o un mismo grupo) en un espacio en sí mismo en movimiento y sometido a incesantes transformaciones" (Bourdieu, 1997b, p. 82). Por esta razón, el autor discute con el pensamiento sustancialista que conduce a tratar las propiedades de los individuos o grupos en un momento puntual del tiempo, como características necesarias e intrínsecas, inscriptas de una vez y para siem- 
pre (Bourdieu, 1997a). La importancia de una mirada diacrónica radica en que la posición actual de un agente no se puede comprender sino a través de la historia estructural de esa posición dentro del espacio social, y de las múltiples disposiciones adquiridas y acumuladas a lo largo de su trayectoria biográfica. De este modo, individuos que ocupan posiciones semejantes en un momento dado, pueden trazar trayectos diferentes en el curso del tiempo (Bourdieu, 1988). La posición de origen es el punto de partida de una trayectoria, el hito con respecto al cual se delinea tanto la pendiente de la trayectoria social como también los caminos posibles de ser recorridos.

A un volumen determinado de capital heredado corresponde un haz de trayectorias más o menos equiprobables que conducen a unas posiciones más o menos equivalentes [...] no siendo igualmente probables todas las posiciones de llegada para todos los puntos de partida (Bourdieu, 1988, p. 108-9).

Estos puntos de llegada condensan en forma de posiciones la trayectoria del grupo social de procedencia. El origen social determina el campo de posibilidades objetivamente ofrecido a un agente, influencia que convierte a una trayectoria individual en un fenómeno de clase: cada clase social está definida por una serie de trayectorias de clase particulares, que toman como punto de origen la pertenencia familiar. Desde esta perspectiva, "la trayectoria modal forma parte integrante del sistema constitutivo de la clase" (Bourdieu 1988, p. 109), al configurar destinos típicos para una misma condición social. En la medida que los agentes siguen los cursos posibles de acción dispuestos para su clase, las trayectorias reproducen el sistema de relaciones dominante.

En contraposición a esta larga tradición sociológica que subraya el peso de los condicionamientos estructurales sobre los individuos, la orientación subjetivista pone énfasis en el proceso de individualización de la vida social y en el carácter yo-yo de las trayectorias. Este paradigma inter- 
pretativo se inscribe en las discusiones sobre la posmodernidad, la crisis de los marcos de referencia colectivos y la capacidad de reflexividad de los individuos. En este sentido, da cuenta de la perspectiva de los sujetos con atributos de agencia y reflexividad, quienes intervienen activamente tanto en la construcción de sus itinerarios como también en la evaluación e interpretación de su contexto objetivo. ${ }^{4}$

Desde esta aproximación, las experiencias biográficas manifiestan la primacía que adquiere el proceso de individualización, presente en la creciente heterogeneidad que revelan los itinerarios contemporáneos. El aporte de la perspectiva subjetivista al análisis de las trayectorias se halla así en la revalorización de la dimensión biográfica que trasluce la complejidad de los rumbos y pasajes que, lejos de ser lineales, son fluctuantes, personalizados e imprevisibles: "lo que hay que describir ya no son únicamente identidades colectivas relativamente estables, sino también trayectorias individuales y sus variaciones en el tiempo" (Fitoussi; Rosanvallon, 1997, p. 31). En este escenario, se inauguran trayectorias que escapan a su estandarización.-

La consecuencia agregada de esta creciente desestructuración social es que las trayectorias contemporáneas ya no pueden determinarse con suficiente certeza. En este sentido, un conjunto de estudios inscriptos en esta perspectiva señala que el itinerario futuro ya no se presenta como un destino último, seguro y previsible; fuera éste la reproducción del origen de clase heredado o la carrera profesional correspondiente a los méritos académicos acumulados (Gil Calvo, 2009). Incluso, la contingencia y los

\footnotetext{
${ }^{4}$ Numerosas perspectivas de análisis han surgido acerca de la sociedad contemporánea, que se focalizaron sobre distintas dimensiones a la hora de estudiarla. Se habla de una sociedad del "riesgo" (Beck, 2006), de la "incertidumbre" (Castel, 2010), "líquida" (Bauman, 2002). Estas diversas aproximaciones arriban a un mismo punto de coincidencia, que enfatiza la autonomía del individuo y el repliegue sobre sí mismo como aspecto clave de la modernidad. Vivimos en una sociedad de individuos (Elias, 1990), en la cual "la capacidad de conducirse como un actor social responsable es cada vez más requerida y cada vez más valorizada" (Castel, 2010: 27).
} 
sucesos imprevisibles se convierten en dimensiones fundamentales para el análisis de trayectorias (Bidart, 2006; Longo, 2010). En este marco, Pais (2007) señala que en la época actual se produce el pasaje desde formas lineales de transición hacia transiciones reversibles y laberínticas.

Frente a estructuras sociales cada vez más fluidas y modeladas en función de los individuos y sus deseos, los jóvenes sienten su vida marcada por crecientes inconstancias, fluctuaciones, discontinuidades, reversibilidades, movimientos auténticos de vaivén [...]. El recurso a la metáfora del yo-yo ayuda a expresar estos movimientos oscilatorios y reversibles (2007, p. 26).

Poner el eje en la perspectiva subjetivista, no significa asentir que la misma sea independiente de la condición social de los sujetos. Por el contrario, este proceso global tiene efectos contrastantes. En efecto, el proceso de individualización amenaza a la sociedad a una contradicción ingobernable "entre quienes puedan asociar el individualismo y la independencia, porque su posición social está asegurada, por un lado, y por el otro quienes lleven su individualidad como una cruz, porque ella significa falta de vínculos y ausencia de protecciones" (Castel, 1995, p. 477). De este modo, la individualización institucionalizada supone un nuevo factor de riesgo y vulnerabilidad en el proceso de construcción biográfica.

Por esta razón, Ulrich Beck (2006) designa como "sociedad del riesgo" a la sociedad moderna. Estas sociedades están constituidas sobre el terreno fértil de la inseguridad porque los individuos no encuentran, ni en ellos mismos ni en su medio inmediato, la capacidad de asegurar su protección, al estar ausente todo tipo de regulación colectiva. Sobre este estado de vulnerabilidad, se despliegan los llamados cada vez más exigentes al dinamismo personal. En este marco, se señala la "ambigüedad" del individualismo moderno: al mismo tiempo que valoriza al individuo, promueve su desafiliación (Castel, 2010). 
Es a la vez un vector de emancipación de los individuos, que incrementa su autonomía y hace de ellos sujetos portadores de derechos, y un factor de inseguridad, que hace a cada uno más responsable de su porvenir y lo obliga a dar a su vida un sentido que ya no organiza nada exterior a sí mismo (Fitoussi; Rosanvallon, 1997, p. 36-7).

En definitiva, el estudio de las trayectorias ha sido abordado desde distintas aproximaciones interpretativas que otorgaron una orientación particular a cada investigación. Existen múltiples usos y aplicaciones, reflejo de las diversas perspectivas teóricas desde las cuales se ha formulado. Un conjunto de estudios toma como eje alguno de estos marcos referenciales, en tanto que otras investigaciones buscan dar cuenta de su articulación; situándose en una encrucijada entre el individuo y la sociedad, entre lo estructural y lo biográfico, lo objetivo y lo subjetivo. Tales conceptualizaciones son diversas, pero cada vez más tendientes a atender la importancia que cobra el sujeto en la construcción de la trayectoria a través de sus decisiones, estrategias y lógicas de acción, en el marco de constreñimientos económicos, sociales y culturales.

Desde este último modelo interpretativo, investigaciones recientes sobre "trayectorias laborales" proponen integrar el rescate de lo biográfico y lo estructural, es decir, las subjetividades de los individuos con los condicionantes estructurales en los que están inmersos. De este modo, el estudio de las trayectorias laborales permite aprehender la imbricación entre el aspecto objetivo y las concepciones subjetivas del trabajo; analiza las posiciones que ocupan los sujetos en el mercado de trabajo, así como las lógicas y sentidos que orientan su recorrido. Esta perspectiva concibe el análisis de los itinerarios ocupacionales como secuencias objetivas y medibles, que atienden también a las construcciones subjetivas que desarrollan los individuos al interior de la matriz de relaciones sociales y estructurales en que viven (Pries, 1996). 
Desde una posición similar, Guzmán, Mauro y Araujo (1999) definen a la trayectoria laboral como los itinerarios visibles, los cursos de acción y las orientaciones que toma la vida de las personas en el campo del trabajo, producto de acciones y prácticas que se constituyen en el interjuego con diferentes ámbitos sociales e institucionales. Si bien los procesos "objetivos" poseen un papel importante en la estructuración de los campos posibles de ser recorridos, la trayectoria laboral permite observar también la manera en que los individuos mediatizan y otorgan sentidos al efecto de las estructuras. Así, las trayectorias laborales proporcionan información sobre las decisiones de los individuos, su capacidad de interpretar las oportunidades y desarrollar estrategias de empleo, trasluciendo una relación subjetiva con el trabajo (Dombois, 1998).

Por esta razón, Márquez (2001) entiende la trayectoria de trabajo no sólo como una categoría objetiva y mensurable, sino como una experiencia social que se construye en relación con la cultura, el mercado y la subjetividad de cada individuo.

Los estudios de las trayectorias laborales abren entonces nuevas posibilidades para la comprensión del mundo del trabajo. No sólo hacen visibles las oportunidades que una determinada época ofrece a hombres y mujeres, sino también las relaciones que existen entre cambios socioeconómicos y culturales, por una parte, y transformaciones de las subjetividades personales y sociales, por la otra. Posibilitan analizar el sentido que estos cambios van teniendo y relacionar entre sí las posiciones que las personas van ocupando en el mercado de trabajo (Mauro, 2004, p. 9).

La perspectiva de las trayectorias laborales posibilita así un análisis fértil para aprehender las transformaciones en el mundo del trabajo. A continuación, señalamos que el enfoque escogido brinda también la posibilidad de analizar estos nuevos fenómenos desde su complejidad analítica, al atender la dimensión objetiva y subjetiva de la realidad social en su articulación espaciotemporal. 


\subsection{La dimensión témporo-espacial}

\section{Multiplicidad de temporalidades: el tiempo histórico, social y biográfico}

Las investigaciones sobre trayectorias encuentran sus raíces en el campo de estudios biográficos. Al interior de este enfoque se presta atención a la interpretación de los fenómenos sociales que se desarrollan en el transcurso del tiempo. De allí, se desprende la importancia de la dimensión temporal para comprender los procesos de cambio y acceder a la forma en que los individuos se adaptan a un ámbito social variable, recuperando aquellas transiciones en los rumbos de una vida. Esta perspectiva teórico-metodológica aporta la temporalidad necesaria para romper con la noción estática de ciertos abordajes sociales; brinda una centralidad substancial al aspecto temporal, al intentar dar seguimiento a lo largo del tiempo a una variedad de procesos. El análisis longitudinal nos remite así a la dimensión diacrónica, y nos sumerge en un camino donde el tiempo individual, social y macroestructural se revelan como partes constitutivas de un itinerario particular. ${ }^{5}$

\footnotetext{
${ }^{5}$ Más allá que supere los objetivos del presente artículo, es importante señalar que la dimensión temporal como dispositivo analítico característico de los estudios con trayectorias, adquiere también otros sentidos vinculados a la estructura diacrónica del relato biográfico-narrativo (Bertaux, 1997; Verd, 2006; Leclerc-Olive, 2009; Schütze; 2010). En este marco, numerosas investigaciones prestan atención a la dimensión cronológica -pasado, presente y futuro- con la finalidad de vislumbrar el modo en que se articulan las distintas escalas de temporalidad en el transcurso de las vidas individuales. Desde esta mirada, la potencialidad de los estudios con trayectorias reside en que el tiempo presente no está determinado sólo por las experiencias acumuladas del pasado del sujeto, sino que también forman parte de él las aspiraciones y los planes para el futuro. Entre presente y futuro, entre sueños y decisiones, entre lo ideal y lo posible, los sujetos van configurando y trazando su trayectoria (Casal et al, 2006; Pais, 2007; Dávila León et al, 2008; Longo, 2010). La premisa subyacente es que los eventos de vida del presente se explican por los cursos de acontecimientos anteriores, que abren un nuevo campo de experiencias y planes venideros. La problemática del tiempo consiste, de este modo, en aprehender cómo los campos de experiencia pasados se reflejan en los horizontes de espera futuros (Koselleck, 1990). Así, estas escalas de temporalidad entran en juego en el análisis y en la conformación de las trayectorias, al constituirse el tiempo como un instrumento de orientación en el mundo (Elias, 1989); donde el pasado estructura el presente (Bourdieu, 1991) y prefigura un futuro (Pries, 1999).
} 
Desde esta mirada, la "temporalidad" ocupa un lugar central en el estudio de las trayectorias. Sin embargo, la variable temporal no debe ser concebida como un aspecto uniforme sino como una dimensión múltiple que puede ser estudiada desde distintos niveles: la dimensión estructural, relacionada con el contexto sociohistórico; la social, vinculada con el ciclo de vida; y la individual, referida a la capacidad de agencia del actor. La multiplicidad de temporalidades remite a las diferentes escalas sociales presentes en toda biografía. De este modo, el curso de vida de un sujeto está determinado por una pluralidad de tiempos, concebidos como tiempo histórico, social y biográfico.

Como varios autores han señalado (Godard, 1996; Coninck; Godard, 1998; Dombois, 1998; Elder, 2001), la importancia de tomar en cuenta la dimensión temporal se evidencia en que las trayectorias se encuentran insertas y moldeadas por los tiempos históricos, aquellos procesos que son externos y condicionantes de la acción social. Las temporalidades "externas" o estructurales están siempre presentes en las trayectorias de los sujetos. Toda trayectoria de vida forma parte de contextos histórico-sociales que condicionan su desarrollo. Sin embargo, el impacto de las estructuras sobre el trayecto de un individuo varía, entre otros aspectos, de acuerdo a su ubicación en el espacio social y en una cohorte de referencia, en cuyos marcos se establecen las oportunidades y constreñimientos que configuran las experiencias biográficas. El estudio de trayectorias ofrece así una vía privilegiada para enmarcar los eventos vitales en un contexto sociohistórico específico.

Ninguna trayectoria individual puede ser abstraída de las particulares condiciones sociales, políticas y económicas en las cuales tiene lugar; toda biografía transcurre en una coyuntura espacio-temporal determinada (Frassa; Muñiz Terra, 2004, p. 9).

Existen diferentes maneras de estudiar el efecto de los cambios históricos en el curso de vida individual. Una aproximación al cambio social 
a través del análisis macro estructural, objetivo central de las Ciencias Sociales y, en especial, de un estilo particular de sociología, conlleva una visión de los fenómenos sociales por fuera e independientemente del accionar humano. Otra manera de abordar dicha problemática encuentra como eje de análisis los cambios de pautas, prácticas y normas que ocurren en los cursos de vida de los individuos, concebidos como instituciones sociales. En este punto, los estudios sobre trayectorias realizan importantes aportes conceptuales y metodológicos, al concebir las articulaciones que existen entre los cambios sociohistóricos y las transformaciones en las subjetividades personales y sociales.

Las pautas de las biografías mismas son un indicador o un reflejo central donde se plasman tanto las regularidades, rutinas o "estructuras" sociales, como el cambio social [...]. La biografía es una institución social en el sentido de un sistema de reglas de acción y de expectativas compartidas (Pries, 1996, p. 403).

Desde esta otra mirada, los trayectos individuales se estructuran por un tiempo social, entendido como aquellas formas sociales de organización temporal de la existencia, que aparecen bajo la imagen de sistemas institucionales y pautas culturales que se construyen sobre disciplinas del tiempo; cada sociedad instituye sus rituales de paso, estableciendo secuencias típicas según los ciclos de vida (Godard, 1996). En consecuencia, las biografías nos remiten a la construcción de temporalidades sociales que regulan y pautan la vida del sujeto, donde el sistema de clasificación por edades contribuye a dar forma a las trayectorias individuales (Gleizer, 1997). Al punto que, las biografías de los sujetos responden en gran medida a un proceso de institucionalización del curso de vida. ${ }^{6}$

\footnotetext{
${ }^{6}$ La "institucionalización del curso de vida" implica un conjunto de etapas socialmente previstas y estipuladas, donde "el ciclo de vida representa la secuencia ideal de acontecimientos que los individuos esperan experimentar y de posicionas sociales que esperan ocupar a medida que avanzan a lo largo de la vida" (Moreno Colom, 2009, p. 194).
} 
Por consiguiente, la edad cronológica adquiere un papel fundamental en el análisis, al dar cuenta de la posición del individuo en el estadio del ciclo vital y la historia. Precisamente, no sólo determina los papeles sociales y roles a desempeñar a una determinada edad, también, remite a un año de nacimiento (cohorte) que ubica al individuo en un determinado tiempo histórico-social (Elder, 1993; Jelin, 2006). En consecuencia, posibilita identificar tanto modificaciones en la composición de las cohortes y las subjetividades, como también cambios en las instituciones y los papeles sociales. $^{7}$

Considerar las distintas etapas de la vida del individuo es importante. Sin embargo, no tenemos que perder de vista que la construcción del tiempo es igualmente subjetiva y se encuentra cargada de sentidos que pueden escapar a las cronologías sociales. Poner el eje en la temporalidad biográfica, por contraposición a las temporalidades instituidas e impuestas socialmente, no significa asentir que la misma sea independiente de la condición social de los sujetos. Los individuos elaboran sus nociones de tiempo a partir de percepciones que están situadas en pertenencias sociales, económicas, culturales, étnicas y de género, conformando una subjetividad particular. Desde este lugar, el tiempo biográfico concibe al individuo como agente activo constructor de su historia y su mundo; el análisis se focaliza aquí en los proyectos, estrategias y decisiones de los sujetos en contextos y tiempos específicos (Pries, 1999; Kohli, 2005; Saraceno, 2005).

Más allá que los estudios sociales han puesto su eje en alguno de estos modelos de temporalidad, es importante señalar que la multiplicidad de tiempos descriptos son construcciones teóricas cuyas divisiones analíticas se presentan en la realidad como dimensiones inescindibles y

\footnotetext{
${ }^{7}$ La realización de estudios inter o intracohortes es una manera de analizar el cambio histórico y su incidencia en el curso de vida individual. Un supuesto impulsado por el paradigma del Curso de Vida es que ese conjunto de personas se mueve o transita a lo largo de la historia. Este fenómeno se conoce como "efecto de cohorte": los cambios históricos se traducen en patrones de vida diferenciados para sucesivas cohortes de nacimiento.
} 
articuladas, a partir de las cuales interpretar y analizar las trayectorias. Así, los modelos temporales se desarrollan de manera conjunta, entrelazándose mutuamente: es necesario conectar las biografías individuales no sólo con las características globales de una situación histórica dada, sino también con los patrones y normas sociales y, por último, con los sentidos, estrategias y vivencias subjetivas.

[Así] las trayectorias se van definiendo y construyendo de manera no lineal a través del tiempo, de acuerdo con la experiencia biográfica, el momento del ciclo de vida, las condiciones y oportunidades ofrecidas por el mercado de trabajo, la percepción de los límites y potencialidades personales, y los cambios sociales y culturales (Mauro, 2004, p. 16).

$\underline{\text { La dimensión espacial como esfera constitutiva de las trayectorias }}$

La relación entre espacio y sociedad es un tema clásico de las Ciencias Sociales. El espacio se constituye como un mosaico de relaciones, formas y sentidos que están determinados por el movimiento de la sociedad. La producción del espacio es, de este modo, el resultado de la acción de los hombres que actúan sobre él. Pero el espacio no sólo contiene el movimiento de la sociedad, también es la cristalización de un momento de su encuentro con las relaciones sociales (Santos, 1995). El espacio se presenta como producto en tanto es el reflejo de una sociedad determinada y como condición al existir en sí mismo e imponerse a la sociedad; estructura las relaciones sociales al tiempo que es producto de las mismas. Desde este lugar, el espacio geográfico no sólo es el escenario o el decorado donde se expresan las desigualdades, sino que juega un papel relevante en la estructuración y desarrollo de las injusticias sociales.

Una vasta literatura ha vislumbrado al espacio urbano como síntesis y promotor de la desigualdad social y, en este sentido, como cristalización y reproducción de las tensiones de la estructura social. Desde la socio- 
logía, se encuentran los estudios clásicos de Bourdieu, quien argumenta contra el pensamiento sustancialista acerca de los lugares y propone pensarlos en clave relacional: "sólo es posible romper con las falsas evidencias y los errores inscriptos en el pensamiento sustancialista de los lugares si se efectúa un análisis riguroso de las relaciones entre las estructuras del espacio social y las del espacio físico" (1999, p. 119). El espacio físico expresa el espacio social y -en tanto espacio social reificado - tiene efectos sociales que cobran importancia en la dinámica espacial, apareciendo el espacio como "uno de los lugares donde se afirma y ejerce el poder, y sin duda en la forma más sutil, el de la violencia simbólica" (1999, p. 122).

En este punto, cabe destacar que entre espacio y sociedad no existe una relación de necesaria correspondencia ni de autonomía absoluta. Si bien el espacio geográfico y el social nunca coinciden exactamente, numerosas diferencias asociadas al efecto del espacio geográfico son el resultado de la distancia en el espacio social, es decir, de una desigual distribución de las diferentes especies de capital (Bourdieu, 1990). Pensar los lugares en clave relacional implica, asimismo, aprehender la dinámica espacial desde las configuraciones macro-micro sociales que presenta. En este sentido, es importante evitar la aplicación de una "razón espacial" que confunde las condiciones en que los acontecimientos ocurren con las causas que provocan dichos acontecimientos, al explicarlos por razones localizadas únicamente en un espacio determinado; así como aquellos enfoques que, enfatizando procesos sociales globales, desconocen los efectos que las configuraciones del espacio producen en la vida social (Segura, 2009). Desde un enfoque similar, Harvey (1997) se aleja tanto del "fetichismo de lo espacial" como de aquellas conceptualizaciones que conciben al espacio como mero soporte de las relaciones sociales, proponiendo en su lugar una articulación entre espacio físico y espacio social.

Por consiguiente, el espacio no es sólo un marco, sino una dimensión constitutiva de lo social. La dinámica espacial impregna la vida cotidia- 
na, las representaciones y las prácticas que sostienen los actores para pensarse a sí mismos y a los otros, en relación a su entorno circundante. A su vez, estas prácticas y representaciones contribuyen de diferentes modos a la producción del espacio, el cual es experimentado de modo desigual por quienes lo habitan. Siguiendo a Lefebvre, el espacio no es un mero "contenedor" ni una simple "expresión" de las relaciones sociales, sino un factor constitutivo de ellas. El espacio es producto de la interacción social, al mismo tiempo que, la sociedad se constituye espacialmente. "Para la "experiencia vivida" el espacio no es un simple "marco" [...] El espacio es la morfología social; en ese sentido, el espacio es a lo 'vivido' lo que al organismo vivo es su propia forma, íntimamente ligada a las funciones y estructuras" (2013, p. 149).

Ahora bien, si en el campo de la sociología el estudio de las categorías espaciales se remonta a los comienzos de la disciplina, autores como Harvey (1998) y Giddens (1984) plantean que la teoría social se ha concentrado con más énfasis en el análisis de las categorías temporales. Mientras el tiempo se convirtió en una dimensión originaria de los estudios del cambio social, el espacio se presentó como una variable de análisis subyugada que expresaba la contextualización del fenómeno bajo estudio. De este modo, la problemática del espacio quedó circunscripta a la ubicación espacial del proceso social analizado y, a lo sumo, a la descripción de ese lugar (Muñiz Terra, 2009). ${ }^{8}$

Desde la aproximación de las trayectorias, como observamos precedentemente, se le ha otorgado una primacía a la temporalidad para el estudio de las vivencias y experiencias que un individuo constituye a lo

\footnotetext{
8 En los estudios sociológicos, la dimensión espacial ha sido analizada a partir de dos grandes conceptualizaciones: el "espacio macro", que presta atención al carácter estructurador del espacio como organizador de percepciones y prácticas de actores sociales. Dentro de esta línea analítica se encuentran: Durkheim, Remy, Bourdieu, Foucault y Castells. El "espacio micro", en contraposición, es un producto de las relaciones sociales, de los cambios y trasformaciones que se producen en la sociedad. Giddens y Lindón son autores que se inscriben en esta perspectiva (Muñiz Terra, 2009).
} 
largo de su vida o en un momento determinado. En la comprensión de las experiencias biográficas es necesaria así una perspectiva a largo plazo que permita dar cuenta del interjuego entre vida individual y tiempo histórico-social. De este modo, la trayectoria se presenta como una perspectiva teórico-metodológica que centra su atención en la interpretación de los fenómenos sociales a lo largo del tiempo, brindado una centralidad substancial al aspecto temporal y, concretamente, a la dimensión cronológica (Roberti, 2015).

Sin embargo, toda biografía se encuentra enmarcada no sólo en un tiempo histórico, sino también en un espacio. El curso vital de los sujetos está moldeado por las diversas escalas de temporalidad y los distintos lugares que experimenta cada persona. La importancia de tomar en consideración la dimensión espaciotemporal radica en que permite ubicar al individuo en el contexto en el que desarrolla su vida. En este sentido, tanto el espacio como el tiempo forman parte de la construcción del objeto de investigación y de la interpretación del dato biográfico.

Pese a que el análisis del espacio encuentra su fundamento en la aproximación biográfica, en los estudios con trayectorias la dimensión espacial se presenta desdibujada a causa de la escasa relevancia analítica que se le ha otorgado. En tal sentido, es primordial señalar que, si bien a un nivel teórico se ha enunciado la importancia de investigar el espacio, los estudios sobre trayectorias han priorizado el análisis de la variable temporal. Si en ocasiones existe una mención respecto a un lugar residencial o un ámbito espacial específico - barrio, ciudad o región -, no se le atribuye al espacio una importancia significativa en el desenvolvimiento del trayecto vital de los individuos; el espacio geográfico no es pensado como una dimensión analítica que confluye en la constitución de las trayectorias. De este modo, no se problematiza la importancia que posee la espacialidad en las transiciones y rumbos que toman los sujetos a 
lo largo de sus vidas. En última instancia, en el estudio de las trayectorias persiste el escaso desarrollo que la sociología como disciplina le ha otorgado a esta categoría.

No obstante, el análisis de la espacialidad brinda importantes contribuciones a las investigaciones de trayectorias. Retomando la perspectiva subjetivista de la geografía francesa, Di Meo (1991) sostiene que el espacio debe ser estudiado como experiencia de la espacialidad. Desde esta mirada se articula, por un lado, el espacio de vida que incluye los lugares frecuentados, como la escuela, el trabajo, los ámbitos de ocio, los itinerarios seguidos -en el plano de la materialidad; y, por otro lado, los espacios vividos que dan cuenta de cómo los mismos son significados e imaginados -en el plano de la representación. En esta línea, Lefebvre (1969, 2013) advirtió tempranamente la necesidad de estudiar las articulaciones entre las formas espaciales y las prácticas sociales como dimensiones constitutivas en la producción del espacio. $\mathrm{Si}$, en un primer sentido, el espacio se presenta como un condicionante de la experiencia social, donde "la vida urbana, la sociedad urbana, en una palabra, "lo urbano" no pueden prescindir de una base práctico-sensible, de una morfología" (1969, p. 67); en un segundo sentido, la categoría espacial se configura a partir de las formas de percepción, significación y acción que despliegan los sujetos en y sobre dicho espacio. En definitiva, prácticas y representaciones producen el espacio social y, a su vez, son producidas por ese espacio.

A modo de cierre, sostenemos que, desde la perspectiva de las trayectorias, constituye una dimensión central del análisis aprehender el modo en que se organiza el espacio a la luz de los itinerarios cotidianos de desplazamiento y las estrategias utilizadas en esa movilidad, dando cuenta de los distintos lugares que trascurren en el curso de vida de un individuo. Al mismo tiempo, adquiere relevancia los significados que los individuos atribuyen al espacio donde viven; en tanto el mismo se concibe como mundo de las percepciones de los sujetos que lo constituyen. 


\section{Reflexiones finales}

Existen múltiples discusiones en torno al uso de trayectorias en Ciencias Sociales. Entre las causas de estas disputas se encuentra el hecho de que, en la investigación sociológica, estos estudios se han desarrollado desde diversas orientaciones y perspectivas teóricas. Como señalamos de manera precedente, el análisis de las trayectorias se ha abordado desde aproximaciones estructuralistas y subjetivistas que han otorgado una orientación particular a cada investigación. En el presente artículo partimos de inscribir el estudio de trayectorias al interior del campo de estudios biográficos. Este enfoque lleva implícito un supuesto ontológico respecto de la realidad social que busca integrar dialécticamente lo universal con lo singular (Ferrarotti, 1993).

De este modo, la dualidad y la interacción existente entre estructura y agencia es un punto de partida relevante en la reconstrucción de trayectorias. Consideramos que los factores estructurales conforman la matriz de relaciones objetivas por la cual los sujetos transitan, pero no explican en su totalidad sus particularidades. Las experiencias, sentidos y estrategias subjetivas permiten comprender las singularidades que adquiere cada recorrido. Por consiguiente, el análisis de trayectorias refleja tanto voluntades individuales como condicionantes estructurales e institucionales, que se entrelazan dinámicamente a lo largo del tiempo y el espacio.

Siguiendo los aportes de estudios clásicos en la temática, concebimos las trayectorias como resultantes de interacciones complejas que integran los tiempos históricos, sociales y biográficos. Uno de los análisis más interesantes respecto a estos ejes de investigación, refiere así a la trama que los vincula y a la incidencia de cada temporalidad en el proceso bajo estudio. Precisamente, las trayectorias son algo más que historias vitales personales: son un reflejo de las relaciones que se establecen entre los procesos sociales más amplios y la configuración de subjetividades que se inscriben en estruc- 
turas y contextos determinados. En este punto, consideramos que el análisis de la espacialidad brinda importantes contribuciones a la investigación social, vinculadas al lugar central que adquiere el espacio no sólo como un marco donde actúan y viven los sujetos; sino también como una dimensión clave para dilucidar las prácticas y representaciones que coadyuvan a la configuración de las trayectorias biográficas.

Eugenia Roberti es becaria doctoral del Consejo Nacional de Investigaciones Científicas y Técnicas (CONICET) y docente en la Facultad de Humanidades y Ciencias de la Educación de la Universidad Nacional de La Plata (FaHCE-UNLP). \eugenia.roberti@hotmail.com

\section{Referencias}

1. BALÁN, J.; JELIN, E. La estructura social en la biografía personal. En: CEDES, v. 2, n. 9, 1979.

2. BALÁN, J. et al. El hombre en una ciudad en desarrollo. Movilidad geográfica y social en Monterrey. México: FCE, 1977.

3. BAUMAN, Z. Modernidad líquida. Buenos Aires: Fondo de Cultura Económica, 2002.

4. BECK, U. La sociedad del riesgo: hacia la nueva modernidad. Barcelona: Paidós, 2016.

5. BERDAGUER, L.; ZARAUZA, D. Jóvenes universitarios con estudios de posgrado. Perspectivas en Argentina: trayectorias educativas y laborales de estudiantes de los posgrados de la Facultad de Bellas Artes de la UNLP. II Congresso Internacional IGLU. Florianópolis, 2011.

6. BERTAUX, D. El enfoque biográfico: su validez metodológica, sus potencialidades. Proposiciones, n. 29. Universidad de Costa Rica, 1999.

7. BERTAUX, D. Les récits de vie. París: Nathan Université, 1997.

8. BIDART, C. Crises, décisions et temporalités: autour des bifurcations biographiques. Cahiers Internationaux de sociologie, n. 120, 2006.

9. BLANCO, M. Trabajo y familia. Entrelazamiento de trayectorias vitales. Estudios Demográficos y Urbanos, n. 51. El Colegio de México. pp.447-483, 2002. 
10. BLANCO, M.; PACHECO, E. Trabajo y Familia desde el enfoque del curso de vida: dos subcohortes de mujeres mexicanas. Papeles de Población, n. 38. México. pp.159-193, 2003.

11. BOURDIEU, P. Espacio social y espacio simbólico. En: Razones prácticas, sobre la teoría de la acción. Barcelona: Anagrama, 1997a.

12. BOURDIEU, P. La ilusión biográfica. En: Razones prácticas, sobre la teoría de la acción, Barcelona: Anagrama, 1997b.

13. BOURDIEU, P. Espacio social y génesis de las "clases". En: Sociología y cultura. México: Grijalbo. pp. 281-310, 1990.

14. BOURDIEU, P. La distinción. Criterios y bases sociales del gusto, Madrid: Taurus, 1988.

15. BUENO FARTES, V. Trajetórias educacionais e profissionais de Trabalhadores na industria petroquímica brasileira: um processo multidimensional de aquisiçao de qualificação. Revista Latinoamericana de estudios del Trabajo, año 7, n. 13. Buenos Aires, 2001.

16. CABANES, R. El aporte teórico del enfoque biográfico para el estudio de un medio social dominado: los obreros de San Pablo. Brasil. En: LULLE, T., VARGAS, P.; ZAMUDIO, L. (coords.). Los usos de la historia de vida en las Ciencias Sociales. Colombia: Anthropos-CIDS, 1998.

17. CASAL, J. y otros. Aportaciones teóricas y metodológicas a la sociología de la juventud desde la perspectiva de la transición. Papers de Sociología, n. 79, 2006.

18. CASTEL, R. El ascenso de las incertidumbres: trabajo, protecciones, estatuto del individuo. Buenos Aires: FCE, 2010.

19. CASTEL, R. La metamorfosis de la cuestión social. Buenos Aires: Paidós, 1995.

20. CASTRO, N.; GANDINI, L. La salida de la escuela y la incorporación al mercado de trabajo de tres cohortes de hombres y mujeres en México. En: V Congreso Nacional de la Asociación Mexicana de Estudios del Trabajo, México, 2006.

21. CLOT, Y. La otra ilusión biográfica. Acta Sociológica, n. 56. México: Centro de estudios Sociológicos/FCPyS/UNAM, 2011.

22. CONINCK, F.; GODARD, F. El enfoque biográfico a prueba de interpretaciones. Formas temporales de causalidad. En: LULLE, T., VARGAS, P.; ZAMUDIO, L. (coords.). Los usos de la historia de vida en las Ciencias Sociales II. Colombia: Anthropos/CIDS, 1998.

23. CRAGNOLINO, E. Género, trabajo y familia. Trayectorias laborales de mujeres de origen campesino en el norte de Córdoba, Argentina. Revista Estudios del Hombre, n. 16. México, 2003. 
24. CUTILI, R. Trayectorias laborales precarizadas. Mujeres de la industria pesquera marplatense. 1980-2008. Mimeo, 2008.

25. DÁVILA LEÓN, O.; GHIARDO, F.; MEDRANO, C. Los desheredados. Trayectorias de vida y nuevas condiciones juveniles. Valparaíso: Ediciones CIDPA, 2008 (segunda edición aumentada).

26. DÁVOLOS, P. Después de la privatización: trayectorias laborales de trabajadores con retiro voluntario. Revista Estudios del Trabajo, n. 21. Buenos Aires, 2001.

27. DI MEO, G. Espaces réels, precues, representes, vécus. L'homme, la société, I'espace. Anthropos: Paris, 1991.

28. DOMBOIS, R. Trayectorias Laborales en la perspectiva comparativa de obreros en la industria colombiana y la industria alemana. En: LULLE, T., VARGAS, P.; ZAMUDIO, L. (coords.). Los usos de la historia de vida en las Ciencias Sociales I. Colombia, Anthropos-CIDS, 1998.

29. ELDER, G. Life course: sociological aspects. En: Smelser y Baltes (eds.), International Encyclopedia of the Social and Behavioral Sciences, v.13. Elsevier: Oxford, 2001.

30. ELDER, G. Time, Human Agency and Social Change: Perspectives on the Life Course. Social Psychology Quarterly. v. 57, n. 1, 1994, pp.4-15.

31. ELDER, G. Historia y trayectoria vital. En: MARINAS J.; SANTAMARINA, C. (eds.). La Historia oral: métodos y experiencias. Madrid: Debate, 1993.

32. ELDER, G. et al. The emergence and development of life course theory. En: MORTIMER, J.; SHANAHAN, M. (eds.). Handbook of the Life Course. New York: Kluwer Academic/Plenum Publishers, 2003.

33. ELIAS, N. La sociedad de los individuos: ensayos. Barcelona, Península, 1990.

34. ELIAS, N. Sobre el tiempo. México: FCE, 1989.

35. FERRAROTTI, F. Biografía y Ciencias Sociales. Historia oral e historias de vida. Cuadernos de Ciencias sociales n. 10, Costa Rica: FLACSO, 1988.

36. FERRAROTTI, F. La Historia y lo cotidiano. Barcelona: Península, 1991.

37. FERRAROTTI, F. Sobre la autonomía del método biográfico. En: MARINAS J.; SANTAMARINA, C. (eds.). La historia oral: métodos y experiencias. Madrid: Debate, 1993.

38. FILMUS, D. et al. La transición entre la escuela secundaria y el empleo: un estudio sobre los recorridos de los jóvenes en el Gran Buenos Aires. Revista Estudios del Trabajo, n. 26. Buenos Aires, 2003. 
39. FITOUSSI, J.; ROSANVALLON, P. La nueva era de las desigualdades. Buenos Aires: Manantial, 1997.

40. FORNI, F.; ROLDAN, L. Trayectorias laborales de residentes de áreas urbanas pobres. Un estudio de casos en el conurbano bonaerense. Desarrollo Económico. v. 35, n. 140, 1996.

41. FRASSA, J. El mundo del trabajo en cambio. Trayectorias laborales y valoraciones subjetivas del trabajo en un estudio de caso. En: 7으소. Buenos Aires, 2005.

42. FRASSA, J.; MUÑIZ TERRA, L. Trayectorias Laborales: origen y desarrollo de un concepto teórico-metodológico. En: $4^{\circ}$ Jornadas de Etnografía y Métodos Cualitativos del IDES. Buenos Aires, 2004.

43. FREIDIN, B. Trayectorias laborales, conceptos y valores sobre el trabajo de mujeres migrantes pobres. En: 20 Congreso Internacional de la Latin American Studie Association. México, 1996.

44. FREYTES FREY, A. Trayectorias de expulsión social: los obstáculos a la inserción laboral en jóvenes 'quemeros' del conurbano bonaerense. En: 8o ASET. Buenos Aires, 2007.

45. GAUTIÉ, J. Transition et trajectoires sur le marché du travail. Quatre Pages, n. 59. París: CEE, 2003.

46. GIDDENS, A. La constitución de la sociedad. Buenos Aires: Amorrortu, 1984.

47. GIL CALVO, E. La rueda de la fortuna: giro en la temporalidad juvenil. En: Congreso de Lisboa: "Jóvenes y Rutas". Madrid: Universidad Cumpletense, 2009.

48. GLEIZER, M. Identidad, subjetividad y sentido en las sociedades complejas. México: FLACSO/Juan Pablos Editor, 1997.

49. GOBTLB I.H.; WHEATON, B. Stress and Adversity over the Life Couse. Trajectories and Turning Points. Nueva York: Cambridge University Press, 1997.

50. GODARD, F. El debate y la práctica sobre el uso de las historias de vida en las ciencias sociales. En: Uso de las Historias de Vida en las Ciencias Sociales. Cuadernos del CIDS. Bogotá: Universidad de Externado de Colombia, 1996.

51. GUZMÁN, V; MAURO, A; ARAUJO, K. Trayectorias laborales de mujeres. Cambios generacionales en el mercado de trabajo. Chile, CEM, 1999.

52. HAREVEN, T. Turning Points and Transitions. Perceptions of the Life Course. Journal of family history. v. 13, n. 3. Sage, 1994. 
53. HAREVEN, T.; de GRUYERE, A. La generación de en medio. Comparación de cohortes de ayuda a padres de edad avanzada dentro de una comunidad estadounidense. Desacatos, Revista de Antropología Social, n. 2, México, 1999.

54. HARVEY, D. Urbanismo y desigualdad social. Madrid: Siglo XXI, 1997.

55. IRRAZABAL, R.; OYARZUN, A. Comportamiento de las trayectorias educacionales y laboral en jóvenes estudiantes. Última Década, n. 18, Chile, pp. 199227, 2003.

56. JACINTO, C. La construcción social de las trayectorias laborales de jóvenes: políticas, instituciones, dispositivos y subjetividades, Buenos Aires: Teseo/ IDES, 2010.

57. JELIN, E. La narrativa personal de lo 'invisible'. Historia, memoria y fuentes orales. 2006.

58. KOHLI, M. Le cours de vie comme institution sociale. En: Enquête, Biographie et cycle de vie, 2005.

59. KOSELLECK, R. Le futur passé. Contribution á la semantique des temps historiques. París: EHESS, 1990.

60. LECLERC-OLIVE, M. Temporalidades de la experiencia: las biografías y sus acontecimientos. Revista Iberforum. año IV, n. 8, México, 2009.

61. LEFEBVRE, H. La producción del espacio. Madrid: Capitán Swing, 2013.

62. LEFEBVRE, H. El derecho a la ciudad. Barcelona: Península, 1969.

63. LONGO, M.E. Trayectorias laborales de jóvenes en Argentina. Tesis Doctoral. UBA/Université de Provence, 2010.

64. MÁRQUEZ, F. Trayectoria de vida y trabajo en sujetos pobres. Proposiciones N³2. Chile, 2001.

65. MAUGER, G. La «jeunesse» dans les «âges de la vie». Une «définition préalable». Temporalistes, n. 11, 1989.

66. MAURO, A. Trayectorias laborales en el sector financiero. Recorridos de las mujeres. En: Unidad de Mujer y Desarrollo de la CEPAL n. 59, Chile, 2004.

67. MILLENAAR, V. Incidencias de la capacitación para el trabajo en las trayectorias laborales de mujeres jóvenes de bajos recursos. Tesis de Maestría en Ciencias Sociales. UNGS/IDES, 2012.

68. MIRANDA, A. et al. Educación más trabajo=menor desigualdad. La inserción educativa y laboral de los egresados 2011. En: PÉREZ, P.; Busso, M. (coord.) Tiempos contingentes: inserción laboral de los jóvenes en la Argentina posneoliberal. Buenos Aires: Miño y Dávila, 2014. 
69. MONTES, N. Trayectorias educativas y laborales: un cruce desde la percepción de estudiantes de nivel medio. En: TIRAMONTI, G.; MONTES, N. (comp.) La escuela media en debate: problemas actuales y perspectivas desde la investigación. Buenos Aires: Manantial/FLACSO, 2009.

70. MOREIRA CARDOSO, A. et al. Os deserdados da indústria: Reestrutura $\square$ ao produtiva e trajetórias intersetoriais de trabalhadores demitidos da indústria brasileira. Revista Latinoamericana de estudios del Trabajo, año 7, n. 13. Buenos Aires, 2001.

71. MORENO COLOM, S. Uso del tiempo, desigualdades sociales y ciclo de vida. Política y Sociedad v. 46, n. 3, 2009, pp.191-202.

72. MUÑIZ TERRA, L. Bifurcaciones. Rupturas y continuidades en las trayectorias laborales de los ex trabajadores petroleros. Un estudio a partir de la privatización de la refinería YPF La Plata. Tesis Doctoral en Ciencias Sociales, UBA, 2009.

73. MUÑIZ TERRA, L.; ROBERTI, E. Revisitando la perspectiva biográfica en clave educativa: un estudio de caso de la formación de posgrado en Argentina a partir de programas curriculares. Revista CUHSO. Cultura-Hombre-Sociedad. v. 23, n. 2, Chile, 2013, pp.83-107.

74. OLIVEIRA, O.; ARIZA, M. Transiciones familiares y trayectorias laborales femeninas en el México urbano. En: GÓMEZ, C. (comp.) Procesos sociales, población y familia: Alternativas teóricas y empíricas en las investigaciones sobre vida doméstica. México: FLACSO/Porrúa, 2001.

75. OTERO, A. Procesos de transición a la vida adulta: un estudio cualitativo con jóvenes argentinos. Tesis Doctoral en Ciencias Sociales. FLACSO-Argentina, 2009.

76. PAIS, J.M. Chollos, chapuzas y changas. Jóvenes, trabajo precario y futuro. Barcelona: Antrhopos, 2007.

77. PANAIA, M. Trayectorias profesionales y demandas empresariales de ingenieros en Argentina. Revista Latinoamericana de Estudios del Trabajo. año 13, n. 7, Buenos Aires, 2001.

78. PIÑA, C. Sobre las historias de vida y su campo de validez en las Ciencias Sociales. En: Documento de Trabajo, n. 319, FLACSO-Chile, 1986.

79. PRIES, L. Conceptos de trabajo, mercados de trabajo y proyectos biográficos laborales. México: Mimeo, 1999.

80. PRIES, L. ¿̇nstitucionalización o desinstitucionalización del curso de vida? Biografía y sociedad como un enfoque integrativo e interdisciplinario. Estudios Demográficos y Urbanos. v. 11, n. 2, 1996, pp.395-417. 
81. PUJADAS MUÑOZ, J. El método biográfico: el uso de las historias de vida en ciencias sociales. Cuadernos Metodológicos, n. 5. Madrid: CIS, 1992.

82. ROBERTI, E. La nueva condición juvenil en tiempos de desestructuración: un estudio de las trayectorias laborales de los jóvenes del barrio Aluvión y su imbricación con otras esferas vitales. Tesis de Maestría en Ciencias Sociales. UNLP, 2015.

83. SALVIA A.; CHAVEZ MOLINA, E. (comp.). Sombras de una marginalidad fragmentada. Aproximaciones a la metamorfosis de los sectores populares de la Argentina. Buenos Aires: Miño y Dávila, 2007.

84. SANTOS, M. La metamorfosis del espacio habitado. Barcelona: Oikos-tau, 1995.

85. SARACENO, C. The time structure of biographies. En: Enquête, Biographie et cycle de vie, 2005.

86. SCHÜTZE, F. Biography Analysis on the Empirical Base of Autobiographical Narratives: How to Analyse Autobiographical Narrative Interviews-Part I, 2010.

87. SEGURA, R. Si vas a venir a una villa, loco, entrá de otra forma. Distancias sociales, límites espaciales y efectos de lugar en un barrio segregado del Gran Buenos Aires. En: GRIMSON, A., FERRAUDI CURTO, M. C.; SEGURA, R. (comp.). La vida política en los barrios populares de Buenos Aires. Buenos Aires: Prometeo. pp.41-62, 2009.

88. SENDÓN, M.A. Las trayectorias de los egresados de la escuela media en una sociedad mutada. Revista Mexicana de Investigación Educativa, v. 10, n. 24. México, 2005, pp. 191-219.

89. TESTA, J.; FIGARI, C. Perfiles, trayectorias y senderos profesionales. Cambios tecnológicos y transformaciones de los perfiles de los técnicos químicos, v. 3, n. 14. Serie Informes de investigación/Ceil-Piette, 2005.

90. TUIRÁN, R. Dominios institucionales y trayectoria de vida en México. En: FIGUEROA, B. (coord.) México Diverso y Desigual. Enfoques sociodemográficos. México, 1999.

91. VERD, J.M. La construcción de indicadores biográficos mediante el análisis reticular del discurso. Una aproximación al análisis narrativo-biográfico. REDESRevista hispana para el análisis de redes sociales, v. 10, n. 7. España, 2006.

92. WRIGHT MILLS, Ch. La imaginación sociológica. México: FCE, 1994.

Recibido: 30.03.2016

Aceito: 24.08.2016 